\title{
Asymptotic formulas for integer partitions within the approach of microcanonical ensemble
}

\author{
D. Prokhorov, A. Rovenchak \\ Department for Theoretical Physics, Ivan Franko National University of Lviv, \\ 12 Drahomanov Str., 79005 Lviv, Ukraine
}

Received May 5, 2012, in final form July 9, 2012

The problem of integer partitions is addressed using the microcanonical approach which is based on the analogy between this problem in the number theory and the calculation of microstates of a many-boson system. For ordinary (one-dimensional) partitions, the correction to the leading asymptotic is obtained. The estimate for the number of two-dimensional (plane) partitions coincides with known asymptotic results.

Key words: integer partitions, plane partition, bosonic systems

PACS: 05.30.Ch, 05.30.Jp

\section{Introduction}

In this paper, we address the problem of integer partitions using the physical approach based on microcanonical treatment. Partitioning of integers is a problem in the number theory that originated in the works by Leibniz [1] and Euler [2]. A partition of a positive integer $n$ is a way of writing $n$ as a sum of positive integers, where the order of the summands is insignificant. The number of partitions $p(n)$ is called a partition function [3, Ch. 1] Further in this work we refer to $p(n)$ simply as the number of partitions to avoid confusion with the respective physical term.

To clarify the notion of partitions, let us consider the number 5. It can be represented as the following sums:

$$
5=4+1=3+2=3+1+1=2+2+1=2+1+1+1=1+1+1+1+1 .
$$

Therefore, one has for the number of partitions $p(5)=7$.

Generalization for higher-dimensional partitions is made in the following fashion. In the $D$-dimensional case, an integer $n$ is represented as a sum of positive integers $n_{i_{1} \ldots i_{D}}$ :

$$
n=\sum_{i_{1}, \ldots, i_{D} \geqslant 0} n_{i_{1} \ldots i_{D}}
$$

where $n_{i_{1} \ldots i_{D}} \geqslant n_{j_{1} \ldots j_{D}}$ whenever $i_{1} \leqslant j_{1}, i_{2} \leqslant j_{2}, \ldots, i_{D} \leqslant j_{D}[3$, p. 179].

For instance, in the case of two-dimensional (plane) partitions of 3 , one has the following possibilities:

$\begin{array}{cccccc}3 & 2+1 & 2 & 1+1+1 & 1+1 & 1 \\ & + & & + & + \\ & 1 & & 1 & 1 \\ & & & & + \\ & & & & & \\ & & & & & \end{array}$

yielding the number of plane partitions $p_{2}(3)=6[3,4]$. This can be easily shown by considering the numbers $n_{i_{1} i_{2}}$ to be elements of square $1 \times 1,2 \times 2,3 \times 3$, and so on, matrices. To obtain the sum of matrix 
elements equal to 3 (provided that they are sorted in a non-ascending order in both rows and columns, according to the above definition), we have:

$$
\text { (3), }\left(\begin{array}{ll}
2 & 1 \\
0 & 0
\end{array}\right),\left(\begin{array}{ll}
2 & 0 \\
1 & 0
\end{array}\right),\left(\begin{array}{ll}
1 & 1 \\
1 & 0
\end{array}\right), \quad\left(\begin{array}{lll}
1 & 1 & 1 \\
0 & 0 & 0 \\
0 & 0 & 0
\end{array}\right), \quad\left(\begin{array}{lll}
1 & 0 & 0 \\
1 & 0 & 0 \\
1 & 0 & 0
\end{array}\right) \text {. }
$$

Note that zero elements are skipped when writing multidimensional partitions.

\section{Physical analogy}

There is a straight analogy between the number of partitions and the number of microstates of a many-boson system of harmonic oscillators. The approach based on such an analogy was suggested as early as in 1950 by Nanda [5], whose paper is now frequently overlooked, and was later utilized by a number of authors to solve related problems [6-8].

Let the system of one-dimensional harmonic oscillators with spectrum $\varepsilon_{j}=\hbar \omega j$ have an energy $E$ :

$$
E=\hbar \omega \sum_{i} j_{i}
$$

where $j_{i}$ denotes the quantum number of the $i$ th particle. The summation runs only over the excited states and thus the particles in the ground state (with zero energy) can be arbitrary in number. The set $\left\{j_{1}, j_{2}, \ldots\right\}$ corresponds to a certain microstate of the system. In the quantum case, the particles are indistinguishable, so the permutation of $\left\{j_{1}, j_{2}, \ldots\right\}$ does not lead to a new microstate. At this point, we can match the set $\left\{j_{1}, j_{2}, \ldots\right\}$ and a partition of the number $n=E / \hbar \omega$. Thus, the number of microstates $\Gamma(E)$ is equal to the number of partitions of $n$. In the set $\left\{j_{1}, j_{2}, \ldots\right\}$, any of the numbers $j_{i}$ can be equal, so one must consider bosonic oscillators.

To make an asymptotic estimation of the number of microstates $\Gamma(E)$, one can use the well-known Hardy-Ramanujan formula [9] for integer partitions:

$$
p^{\mathrm{HR}}(n)=\frac{1}{4 \sqrt{3} n} \mathrm{e}^{\pi \sqrt{2 / 3} \sqrt{n}}
$$

therefore,

$$
\Gamma(E)=\frac{1}{4 \sqrt{3} E / \hbar \omega} \mathrm{e}^{\pi \sqrt{2 / 3} \sqrt{E / \hbar \omega}}
$$

In the next section, we will derive this expression from physical considerations and obtain the first correction to this asymptotic result. For simplicity, we further put the unit of energy $\hbar \omega=1$.

The above considerations might be extended - with minor reservations - to higher-dimensional partitions, the two-dimensional (plane) ones being best studied. The respective analysis is presented in section 4

\section{Corrections to the leading asymptotics of $p(n)$}

Partition function $Z(\beta)$ can be expressed as an integral by introducing the number of microstates $\Gamma(E)$ :

$$
Z(\beta)=\sum_{j} \mathrm{e}^{-\beta E_{j}}=\int_{0}^{\infty} \Gamma(E) \mathrm{e}^{-\beta E} \mathrm{~d} E .
$$

The above expression is nothing but the Laplace transform, so, by inverting it, we obtain:

$$
\Gamma(E)=\frac{1}{2 \pi \mathrm{i}} \int_{\gamma-\mathrm{i} \infty}^{\gamma+\mathrm{i} \infty} Z(\beta) \mathrm{e}^{\beta E} \mathrm{~d} \beta
$$


The entropy $S(\beta)$ is equal to

$$
S(\beta)=\beta E+\ln Z(\beta) .
$$

Thus, we have

$$
Z(\beta)=\mathrm{e}^{S(\beta)} \mathrm{e}^{-\beta E}
$$

and

$$
\Gamma(E)=\frac{1}{2 \pi \mathrm{i}} \int_{\gamma-\mathrm{i} \infty}^{\gamma+\mathrm{i} \infty} \mathrm{e}^{S(\beta)} \mathrm{d} \beta .
$$

Using the Taylor series for entropy in the vicinity of $\beta_{0}$ :

$$
S(\beta) \simeq S\left(\beta_{0}\right)+\frac{1}{2 !} S^{\prime \prime}\left(\beta_{0}\right)\left(\beta-\beta_{0}\right)^{2}+\frac{1}{3 !} S^{\prime \prime \prime}\left(\beta_{0}\right)\left(\beta-\beta_{0}\right)^{3},
$$

where $\beta_{0}$ is the stationary point,

$$
S^{\prime}\left(\beta_{0}\right)=0
$$

for the number of microstates, one obtains:

$$
\Gamma(E) \simeq \frac{\mathrm{e}^{S\left(\beta_{0}\right)}}{2 \pi \mathrm{i}} \int_{\gamma-\mathrm{i} \infty}^{\gamma+\mathrm{i} \infty} \exp \left[\frac{1}{2 !} S^{\prime \prime}\left(\beta_{0}\right)\left(\beta-\beta_{0}\right)^{2}+\frac{1}{3 !} S^{\prime \prime \prime}\left(\beta_{0}\right)\left(\beta-\beta_{0}\right)^{3}\right] \mathrm{d} \beta .
$$

Using the replacement $\beta=\mathrm{i} x+\beta_{0}$, we get:

$$
\begin{aligned}
\Gamma(E) & \simeq \frac{\mathrm{e}^{S\left(\beta_{0}\right)}}{2 \pi} \int_{-\infty}^{\infty} \exp \left[-\frac{1}{2 !} S^{\prime \prime}\left(\beta_{0}\right) x^{2}-\frac{1}{3 !} S^{\prime \prime \prime}\left(\beta_{0}\right) \mathrm{i} x^{3}\right] \mathrm{d} x \\
& =\frac{\mathrm{e}^{S\left(\beta_{0}\right)}}{2 \pi} \int_{-\infty}^{\infty} \mathrm{e}^{-\frac{1}{2 !} S^{\prime \prime}\left(\beta_{0}\right) x^{2}}\left\{\cos \left[\frac{S^{\prime \prime \prime}\left(\beta_{0}\right)}{3 !} x^{3}\right]-\mathrm{i} \sin \left[\frac{S^{\prime \prime \prime}\left(\beta_{0}\right)}{3 !} x^{3}\right]\right\} \mathrm{d} x \\
& =\frac{\mathrm{e}^{S\left(\beta_{0}\right)}}{2 \pi} \int_{-\infty}^{\infty} \mathrm{e}^{-\frac{1}{2 !} S^{\prime \prime}\left(\beta_{0}\right) x^{2}} \cos \left[\frac{S^{\prime \prime \prime}\left(\beta_{0}\right)}{3 !} x^{3}\right] \mathrm{d} x .
\end{aligned}
$$

This integral can be expressed via the modified Bessel function of the second kind, namely:

$$
\Gamma(E) \simeq \frac{\mathrm{e}^{S\left(\beta_{0}\right)}}{2 \pi} \frac{2 S^{\prime \prime}\left(\beta_{0}\right)}{\sqrt{3}\left|S^{\prime \prime \prime}\left(\beta_{0}\right)\right|} \exp \left\{\frac{\left[S^{\prime \prime}\left(\beta_{0}\right)\right]^{3}}{3\left[S^{\prime \prime \prime}\left(\beta_{0}\right)\right]^{2}}\right\} K_{1 / 3}\left(\frac{\left[S^{\prime \prime}\left(\beta_{0}\right)\right]^{3}}{3\left[S^{\prime \prime \prime}\left(\beta_{0}\right)\right]^{2}}\right) .
$$

To calculate the entropy $S(\beta)$, we write the partition function of the oscillator system as follows:

$$
Z(\beta)=\prod_{j=1}^{\infty}\left(1-\mathrm{e}^{-\beta j}\right)^{-1}, \quad \ln Z(\beta)=-\sum_{j=1}^{\infty} \ln \left(1-\mathrm{e}^{-\beta j}\right) .
$$

Using the Euler-Maclaurin formula to calculate the sum, one obtains [7]:

$$
S(\beta)=\beta E+\ln Z(\beta)=\beta E+\frac{\pi^{2}}{6 \beta}+\frac{1}{2} \ln \beta-\frac{1}{2} \ln (2 \pi)+\ldots .
$$

Limiting ourselves to the first two terms, the stationary point yields:

$$
\beta_{0}=\frac{\pi}{\sqrt{6 E}} .
$$


With the same accuracy, we have:

$$
S^{\prime \prime}\left(\beta_{0}\right)=\frac{2 \sqrt{6}}{\pi} E^{3 / 2}, \quad S^{\prime \prime \prime}\left(\beta_{0}\right)=-\frac{36}{\pi^{2}} E^{2} .
$$

Collecting the whole expression (3.9) together, for the number of microstates we obtain

$$
\Gamma(E)=\frac{1}{18 \sqrt[4]{6} E^{3 / 4}} \exp \left(\frac{28}{27} \pi \sqrt{\frac{2 E}{3}}\right) K_{1 / 3}\left(\frac{1}{27} \pi \sqrt{\frac{2 E}{3}}\right) .
$$

From this formula, there follows a correction to the main asymptotics of the number of partitions, as one substitutes $E$ with $n$ :

$$
p(n)=\frac{1}{18 \sqrt[4]{6} n^{3 / 4}} \exp \left(\frac{28}{27} \pi \sqrt{\frac{2 n}{3}}\right) K_{1 / 3}\left(\frac{1}{27} \pi \sqrt{\frac{2 n}{3}}\right) .
$$

Taking into account the asymptotic series expansion for large arguments [10]

$$
K_{v}(z) \propto \sqrt{\frac{\pi}{2}} \frac{\mathrm{e}^{-z}}{\sqrt{z}}\left[1+\mathscr{O}\left(\frac{1}{z}\right)\right]
$$

we immediately arrive at the leading asymptotic behavior given by equation (2.2).

\section{Asymptotic behavior of plane partitions}

We consider the energy spectrum of a 2-dimensional system in the following form:

$$
\varepsilon\left(j_{1}, j_{2}\right)=j_{1}+j_{2} .
$$

To obtain $\Gamma(E)$, we repeat the derivation presented in the previous section following [7], see also [11]. Thus, the entropy $S(\beta)$ is equal to

$$
S(\beta)=\beta E+\ln Z(\beta)
$$

where for energy spectrum (4.1), the partition function is

$$
Z(\beta)=\prod_{\text {all the energies }}\left(1-\mathrm{e}^{-\beta \varepsilon}\right)^{-1}=\prod_{j_{1}=1}^{\infty} \prod_{j_{2}=1}^{\infty}\left[1-\mathrm{e}^{-\beta\left(j_{1}+j_{2}\right)}\right]^{-1} .
$$

For the logarithm of the partition function $Z(\beta)$, one has:

$$
\ln Z(\beta)=-\sum_{j_{1}=1}^{\infty} \sum_{j_{2}=1}^{\infty} \ln \left[1-\mathrm{e}^{-\beta\left(j_{1}+j_{2}\right)}\right]=-\sum_{j=1}^{\infty} g_{j} \ln \left[1-\mathrm{e}^{-\beta j}\right],
$$

where the $j$ th level degeneracy is equal to

$$
g_{j}=j+1 \simeq j
$$

therefore,

$$
\ln Z(\beta)=-\sum_{j=1}^{\infty} j \ln \left[1-\mathrm{e}^{-\beta j}\right]
$$

After applying the Euler-Maclaurin summation formula, the entropy can be expressed in such a form:

$$
S(\beta)=\beta E+\ln Z(\beta)=\beta E+\frac{\zeta(3)}{\beta^{2}}+\frac{1}{12} \ln \beta-\frac{1}{6} .
$$


The standard saddle-point method, for $\Gamma(E)$ from equation (3.1), yields:

$$
\Gamma(E)=\frac{\exp \left[S\left(\beta_{0}\right)\right]}{\sqrt{2 \pi S^{\prime \prime}\left(\beta_{0}\right)}} .
$$

As in the previous section, for the stationary point $\beta_{0}$, so that $S^{\prime}\left(\beta_{0}\right)=0$, one obtains

$$
\beta_{0}=\left[\frac{2 \zeta(3)}{E}\right]^{1 / 3}
$$

and

$$
S^{\prime \prime}\left(\beta_{0}\right)=\frac{3}{[2 \zeta(3)]^{1 / 3}} E^{4 / 3}
$$

Thus, the number of microstates is

$$
\Gamma(E)=\frac{1}{\sqrt{6 \pi}}[2 \zeta(3)]^{7 / 36} E^{-25 / 36} \exp \left\{\frac{3}{2}[2 \zeta(3)]^{1 / 3} E^{2 / 3}-\frac{1}{6}\right\} .
$$

Substituting energy $E$ with integer $n$, we immediately obtain the result for plane partitions in the following way:

$$
p_{2}(n)=\frac{1}{\sqrt{6 \pi}}[2 \zeta(3)]^{7 / 36} n^{-25 / 36} \exp \left\{\frac{3}{2}[2 \zeta(3)]^{1 / 3} n^{2 / 3}-\frac{1}{6}\right\} .
$$

Our estimation differs from the result of Wright [12]

$$
p_{2}^{\mathrm{W}}(n)=\frac{1}{\sqrt{6 \pi}}[2 \zeta(3)]^{7 / 36} n^{-25 / 36} \exp \left\{\frac{3}{2}[2 \zeta(3)]^{1 / 3} n^{2 / 3}+c\right\}
$$

by a constant factor:

$$
c=\zeta^{\prime}(-1)=-0.165421 \ldots \text { versus }-\frac{1}{6}=-0.166666 \ldots
$$

Note that Wright's asymptotics was later confirmed by Nanda [5] with a much more sophisticated analysis of the oscillator system as compared to our approach.

\section{Results and discussion}

We performed calculations for the number of ordinary partitions $p(n)$ and plane partitions $p_{2}(n)$ using the expressions (3.15) and (4.12) obtained in this work.

The comparison with exact values and the leading asymptotics by Hardy and Ramanujan are given in table 1 and in figure 1. In the figure, the relative errors are plotted. For $n=32$ and $n>33$, our correction becomes negative. For $n>20$, the error is less than one per cent, and it never exceeds seven per cent except for $n=1$, where $p(n)=1$, and our formula yields 2, and $n=5$ with our value of 8 versus $p(n)=5$, which leads to about 15 per cent error. Beyond this domain, the maximum relative deviation of 0.0083285 is found for $n=186$.

The plane partitions are demonstrated in table 2 and in figure 2 Our result for plane partitions provides a better estimation compared to that of Wright up to $n=7573$. At $n=2679$, expression (4.12) starts to underestimate the number of plane partitions while (4.13) asymptotically approaches the real values of $p_{2}(n)$ from above for all $n$. Interestingly, the approach to the calculation of plain partitions used in this work which takes into account the level degeneracy provides a much better estimate compared to an earlier suggested [15] direct treatment of multidimensional oscillators which failed to produce a correct pre-exponential behavior of the power of $n$.

To summarize, the expressions for the number of integer partitions were obtained from the analogy between this number-theoretical problem and the physical problem of calculating the states in a manyboson system within the microcanonical approach. The correction to the main asymptotics for ordinary (one-dimensional) partitions is shown to give a good estimate even for small numbers. 


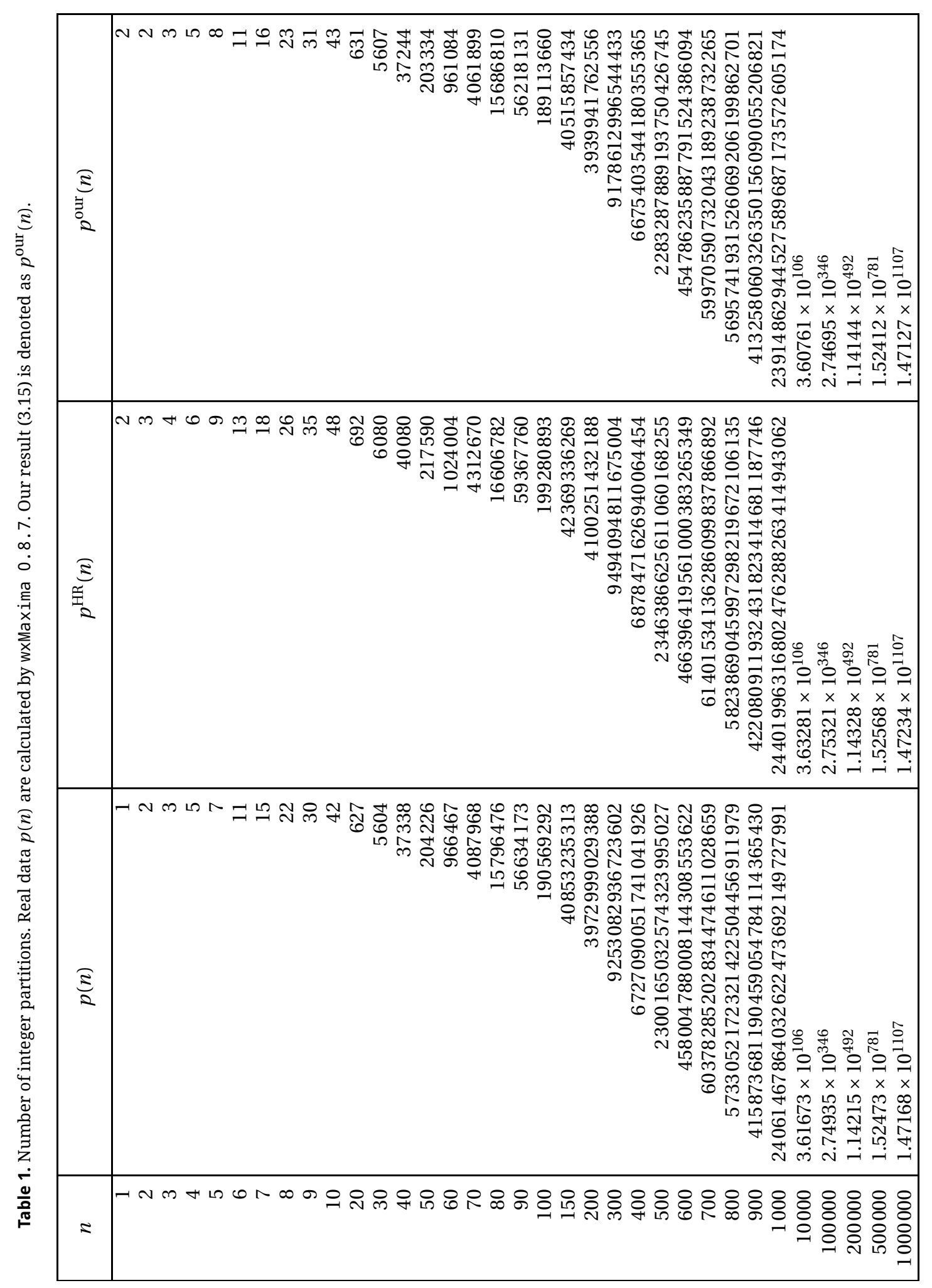




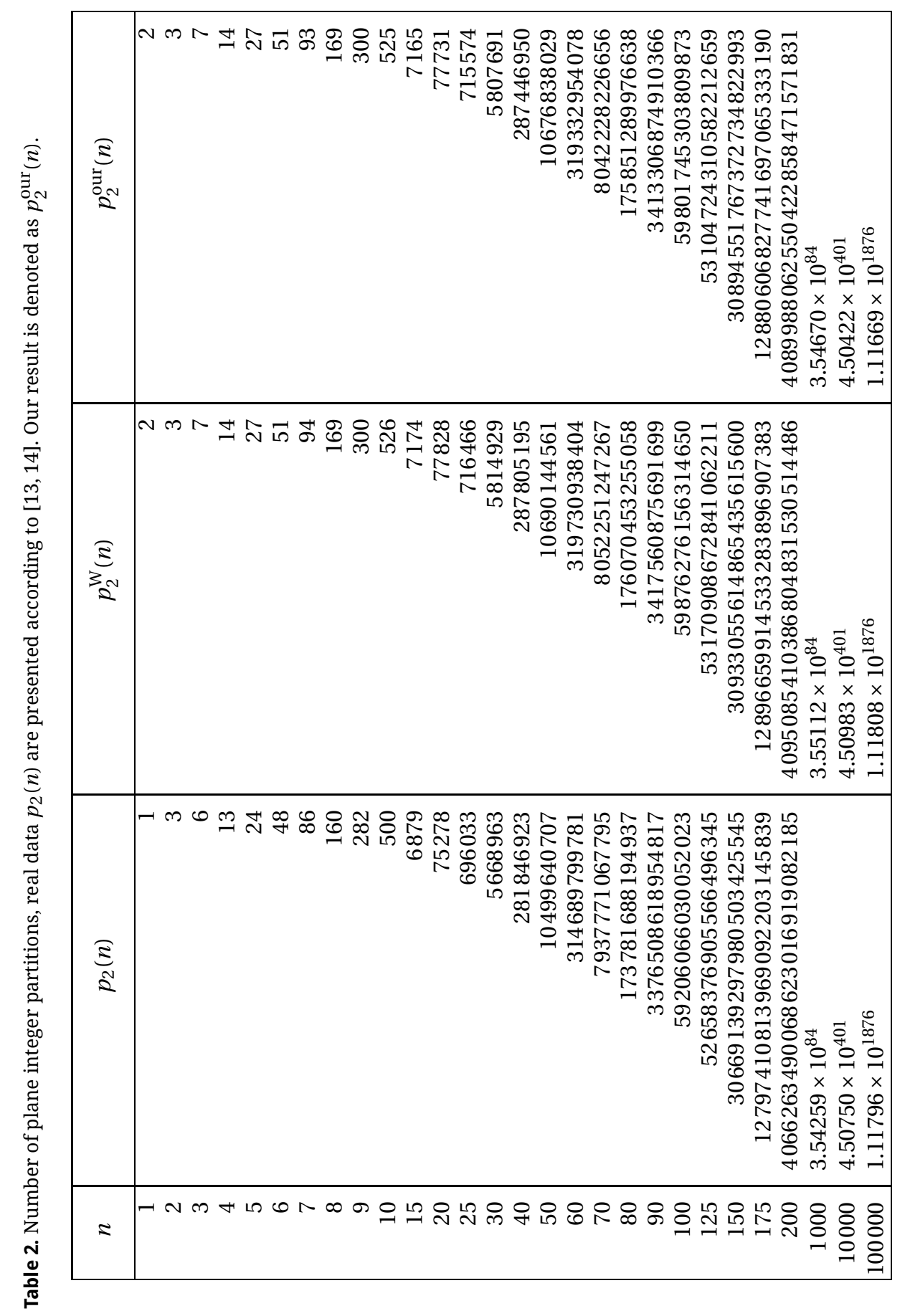



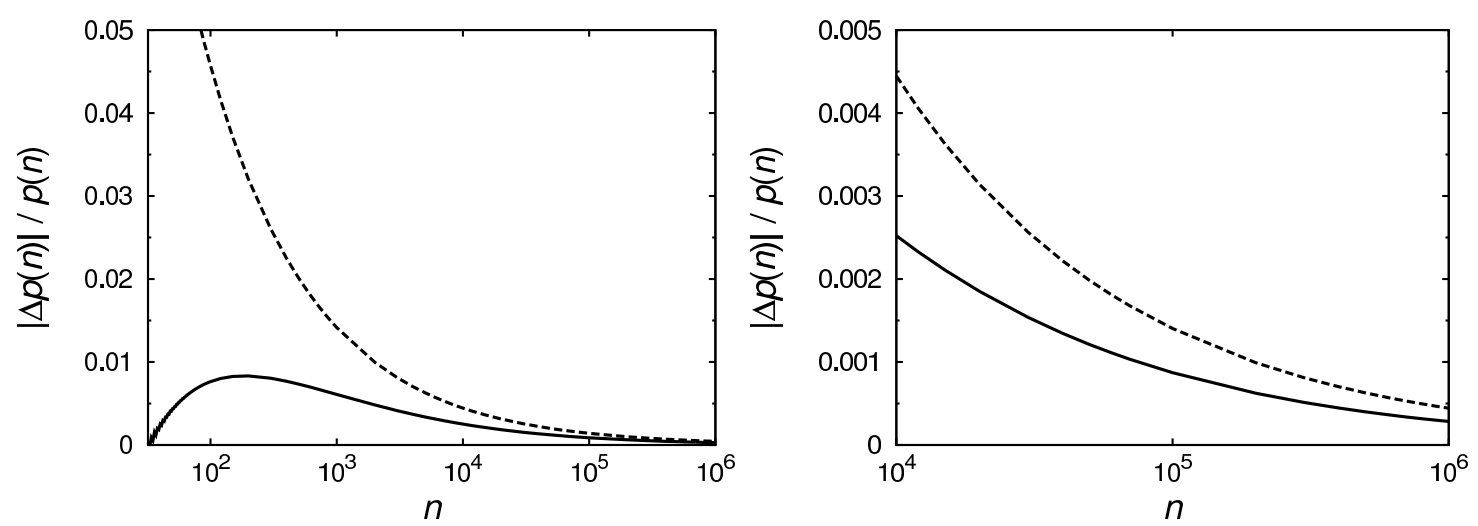

Figure 1. Comparison of relative errors for the estimations of the number of integer partitions from the real values (calculated by wxMaxima 0.8 .7 ). Solid curve - our result [3.15), dashed curve - the leading asymptotics provided by the Hardy-Ramanujan formula (2.2). Large $n$ domain in an enlarged view is presented on the right.

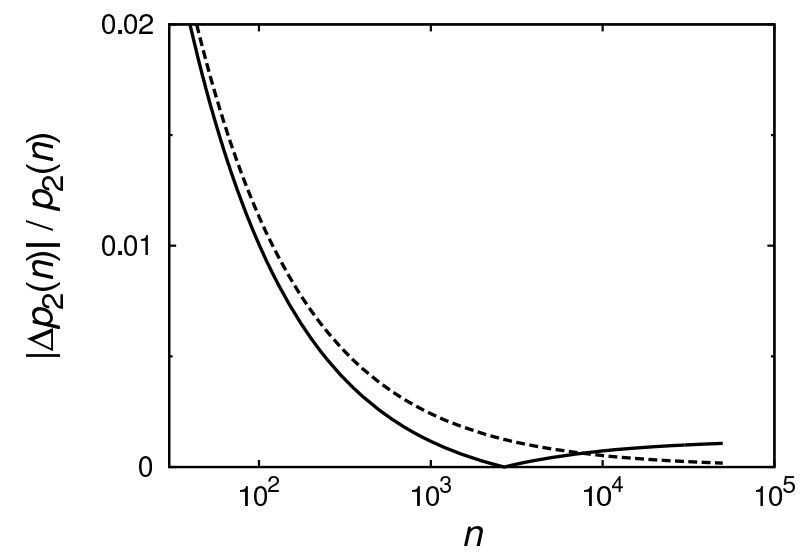

Figure 2. Comparison of relative errors for the estimations of the number of plane partitions from the real values $p_{2}(n)[13,14]$. Solid curve - our result [4.12), dashed curve - Wright's formula [4.13].

\section{Acknowledgements}

We thank Yuri Krynytskyi for discussions on some issues raised in this paper. The work was partly supported by grant $\Phi \Phi-110 \Phi$ (No. 0112U001275) from the Ministry of education, science, youth, and sports of Ukraine.

\section{References}

1. Leibniz G.W. - In: Sämtliche Schriften und Briefe. Siebente Reihe: Mathematische Schriften, Bd. 1: 1672-1676. Geometrie - Zahlentheorie - Algebra (1. Teil). Akademie-Verlag, Berlin, 1990, p. 740-755.

2. Eulero L., Novi Commentarii Academiae scientiarum Petropolitanae, 1753, 3, 125.

3. Andrews G.E., The Theory of Partitions. Addison-Wesley. Reading, MA, 1976.

4. Almkvist G., Experimental Mathematics, 1998, 7, 343; doi 10.1080/10586458.1998.10504380.

5. Nanda V.S., Math. Proc. Cambridge Philos. Soc., 1951, 47, 591; doi 10.1017/S0305004100026980

6. Grossmann S., Holthaus M., Phys. Rev. Lett., 1997, 79, 3557; doi 10.1103/PhysRevLett.79.3557

7. Tran M.N., Murthy M.V.N., Bhaduri R.J., Ann. Phys., 2004, 311, 204; doi 10.1016/j.aop.2003.12.004

8. Comtet A., Leboeuf P., Majumdar S.N., Phys. Rev. Lett., 2007, 98, 070404; doi 10.1103/PhysRevLett.98.070404

9. Hardy G.H., Ramanujan S., Proc. London Math. Soc., 1918, 17, 75; doi 10.1112/plms/s2-17.1.75

10. Abramowitz M., Stegun I.A., Handbook of Mathematical Functions, tenth printing. National Bureau of Standards, 1972. 
11. Rovenchak A., Fiz. Nizk. Temp., 2009, 35, 510 [Low Temp. Phys., 2009, 35, 400; doi 10.1063/1.3132748].

12. Wright E.M., Q. J. Math., 1931, 177; doi 10.1093/qmath/os-2.1.177

13. Mutafchiev L., Kamenov E., Compt. Rend. Acad. Bulg. Sci., 2006, 59, 361.

14. http://oeis.org/A000219/b000219.txt

15. Rovenchak A., Computat. Meth. Sci. Technol. (Poznań), 2010, 16, 187.

\title{
Асимптотичні формули для розбиттів цілих чисел у підході мікроканонічного ансамблю
}

\author{
Д. Прохоров, А. Ровенчак \\ Кафедра теоретичної фізики, Львівський національний університет імені Івана Франка, \\ вул. Драгоманова, 12, Львів, 79005, Україна \\ Розглянуто задачу про розбиття цілих чисел у межах мікроканонічного підходу, який ґрунтується на ана- \\ логії між цією задачею з теорії чисел і обчисленням кількості мікростанів багатобозонної системи. Для \\ звичайних (одновимірних) розбиттів отримано поправку до головної асимптотики. Оцінка кількості дво- \\ вимірних (плоских) розбиттів добре узгоджується з відомими асимптотичними результатами.
}

Ключові слова: розбиття цілих чисел, плоскі розбиття, бозонні системи 


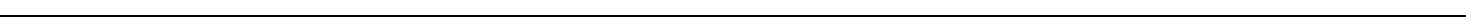

